Www.jmscr.igmpublication.org

Impact Factor (SJIF): 6.379

Index Copernicus Value: 71.58

ISSN (e)-2347-176x ISSN (p) 2455-0450

crossref DOI: https://dx.doi.org/10.18535/jmscr/v6i2.75

\begin{abstract}
IGM Publication
Journal Of Medical Science And Clinical Research

An official Publication of IGM Publication
\end{abstract}

\title{
Retrospective Analysis-Anatomo-Histopathological Data of Cancer Stomach in Patients at Government Thiruvarur Medical College \& Hospital (GTMCH) From February-2017 to December-2017
}

Authors

\author{
Dr T. Srinivasan. M.S. ${ }^{1}$, Dr Jishnu V.Mohan ${ }^{2}$, Dr Kanimozhi. $\mathrm{K}^{3}$ \\ ${ }^{1}$ Associate Professor of Surgery, Govt Thiruvarur Medical College \\ ${ }^{2 \& 3}$ CRRI, Dept of General Surgery, Govt Thiruvarur Medical College
}

\begin{abstract}
Background: Anatomical \& Histopathological evaluation has a major role in the management of carcinoma stomach. It decides the mode of treatment we should follow and the prognosis of the treatment for that patient.

Methods: In this retrospective study, records from 20 patients with gastric cancer admitted between 1 February, 2016 and 28 February 2017 were analyzed. Data regarding patient investigations, type of surgery, anatomical site of cancer and macroscopic and histological findings along with the metastatic character of the cancer were obtained from the records.

Results \& Conclusion: The study shows that men are more affected than women and the chance increases with age. Antropyloric region is the commonly affected site. The commonest histopathological type being Adenocarcinoma, that too moderately differentiated form. These data highlight the urgent need for the implementation of preventive measures and early detection programs of gastric cancer.
\end{abstract}

\section{Introduction}

\section{Purpose of the Project}

This paper investigates the trend, the most common gender and age group affected by $\mathrm{Ca}$ Stomach with the help of data collected from the records of patient admitted within the duration of one year (2016 February to 2017 February) from Department of Pathology, GTMCH. The common anatomical site and the histopathological variety are also analyzed retrospectively in this study.

\section{Background}

Gastric cancer is the fourth most commonly diagnosed cancer, the second most common cause of cancer-related death worldwide and the most common type of cancer in Tamil Nadu. According to Tamil Nadu Cancer Registry Project (TNCRP), a project launched by Tamil Nadu Government in 2013, 53,363 new cancer cases are registered in Tamil Nadu during 2012-2014. Out of this, were 23,149 men and 30,214 women. Cancers of the stomach $(2,202$ cases), mouth $(1,935)$ and lung $(1,934)$ were the most common among men. Among women, breast cancer $(7,931)$ was slightly ahead of cervix cancer $(7,913)$. In accordance with new reports, Tamil Nadu is expected to occupy more than $8 \%$ of all the cancer cases of the country. 
Gastric cancer is rare before the age of 40 , but its incidence steadily climbs thereafter and peaks in the seventh decade of life. Gastric carcinogenesis is a multistep and multifactorial process. Ninety percent of all tumors of the stomach are malignant, and gastric adenocarcinoma comprises $95 \%$ of the total number of malignancies. Curative therapy involves surgical resection, most commonly a total or subtotal gastrectomy, with an accompanying lymphadenectomy. The overall 5year survival rate of patients with resectable gastric cancer ranges from $10 \%$ to $30 \%$.

Gastric cancer may often be multifactorial, involving both inherited predisposition and environmental factors. ${ }^{[12]}$ Environmental factors implicated in the development of gastric cancer include the following:

- Diet

- Smoking

- Helicobacter pylori infection

- Genetic factors

- Previous gastric surgery

- Pernicious anemia

- Adenomatous polyps

- Chronic atrophic gastritis

- Radiation exposure

- Epstein-Barr virus

- Gactric ulcers

- Obesity

- Bisphosphonates etc...

While the intestinal type of gastric cancer is often related to environmental factors such as Helicobacter pylori infection, diet, and life style, the diffuse type is more often associated with genetic abnormalities. Recent advances in molecular medicine have not only shed light on the carcinogenesis of gastric cancer, but also offered novel approaches regarding prevention, diagnosis and therapeutic intervention.

WHO has revised the definition of gastric cancer to "malignant epithelial tumors of the gastric mucosa with glandular differentiation". The WHO system assigns grades to adenocarcinoma based on the degree of resemblance to metaplastic intestinal tissue. It categorizes the histologic patterns into 5 subtypes :
- Adenocarcinoma (Intestinal And Diffuse)
○ Papillary
○ Tubular
- Mucinous
○ Signet-Ring Cell.

Gastric carcinoma often produces no specific symptoms when it is superficial and potentially surgically curable, although up to $50 \%$ of patients may have nonspecific gastrointestinal complaints such as dyspepsia. The lack of early pathognomic symptoms often delays the diagnosis. Patients who present with locally advanced or metastatic tumors have poor rates of resectability. Patients may present with anorexia and weight loss as well as abdominal pain that is vague and insidious in nature. Nausea, vomiting, and early satiety may occur with bulky tumors that obstruct the gastrointestinal lumen or infiltrative lesions that impair stomach distension. Ulcerated tumors may cause bleeding that manifest as hematemesis, melena, or massive upper gastrointestinal hemorrhage.

Physical examination of early gastric cancer is usually uninformative. Patients with advanced tumors may present with a palpable abdominal mass, cachexia, bowel obstruction, ascites, hepatomegaly, and lower extremity edema. Peritoneal seeding may cause involvement of the ovaries (Krukenberg tumor) or pelvic cul-de-sac (Blumer's shelf) detectable on rectal examination. Metastasis may manifest as an enlarged supraclavicular lymph node (Virchow's node), left axillary lymph node (Irish's node), or a periumbilical lymph node (Sister Mary-Joseph's node).

Even though the goul of mass screening or surveillance is detection of the carcinoma at an early and therefore a potentially curable stage, Mass endoscopic or radiological screening is not yet on move in comparatively low incident countries like India. 
Endoscopy is regarded as the most sensitive and specific diagnostic method in patients suspected of harboring gastric cancer. When combined with endoscopy and radiologic modalities, endoscopic ultrasound (EUS) can maximize tumor staging by providing information about depth of tumor invasion and assess the extent of perigastric lymphadenopathy. CT has a sensitivity of $88 \%$ for tumor detection. CT is the most frequently used modality for staging gastric cancer. CT can detect liver metastases, regional and distant lymphadenopathy, and can predict direct invasion of adjacent structures.

The management of gastric cancer continues to evolve. Whilst surgery alone is effective when tumours present early, a large proportion of patients are diagnosed with loco-regionally advanced disease, resulting in high loco-regional and distant relapse rates, with subsequent poor survival. Surgery remains the cornerstone of curative therapy in gastric cancer. Choice of surgical procedure in resectable gastric cancer is dictated by size, location, and ability to achieve surgical margins free of gross and microscopic disease. Prognosis depends on the Site, Grade (well, moderately, or poorly differentiated / anaplastic), Extend, Age, Genetic factors etc. The role of postoperative chemoradiotherapy continues to be debated.

Study Design: Retrospective study.

Methodology: Retrospective study of 20 patients suffering from carcinoma stomach admitted between February, 2016 and December 2017 admitted in GTMCH. Data regarding patient investigations, type of surgery, anatomical site of cancer and macroscopic and histological findings along with the metastatic character of the cancer were obtained from The Department of Pathology, GTMCH.

\section{Gender Distribution}

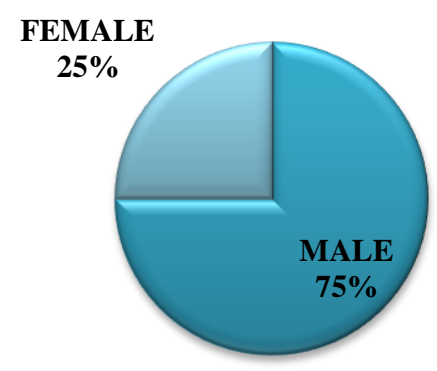

Fig 1: Gender distribution

Table 1 Gender Distribution

\begin{tabular}{|l|c|}
\hline SEX & NUMBER \\
\hline MALE & 15 \\
\hline FEMALE & 5 \\
\hline
\end{tabular}

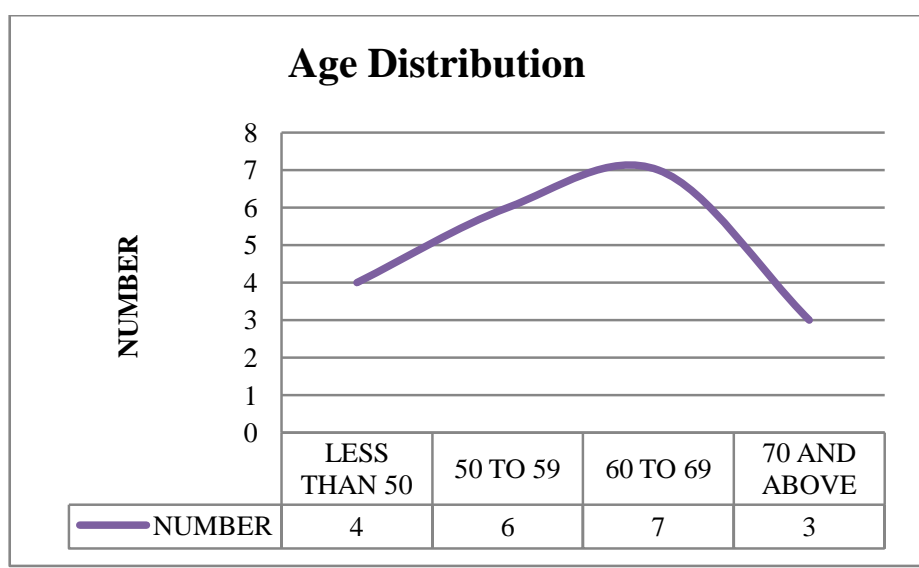

Fig 2: Age distribution

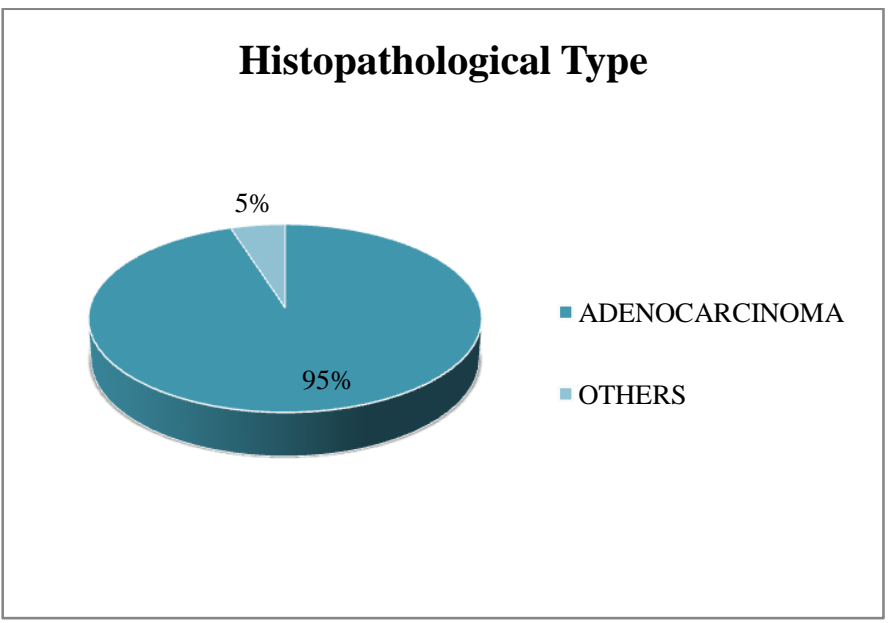

Fig 3: Histopathological type.

Table 2: Histopathological type

\begin{tabular}{|l|c|}
\hline Histopathological Type & Number \\
\hline Adenocarcinoma & 19 \\
\hline Others & 1 \\
\hline
\end{tabular}




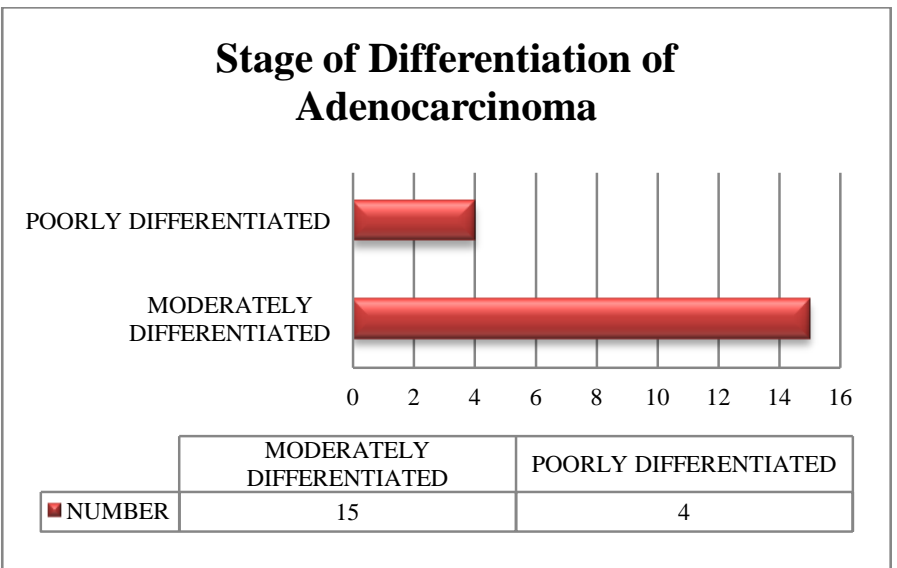

Fig 4: Stage of differentiation of Adenocarcinoma

Table 3: Site of cancer

\begin{tabular}{|l|c|}
\hline SITE & NUMBER \\
\hline OG JUNCTION & 1 \\
\hline BODY AND FUNDUS & 4 \\
\hline ANTROPYLORUS & 17 \\
\hline
\end{tabular}

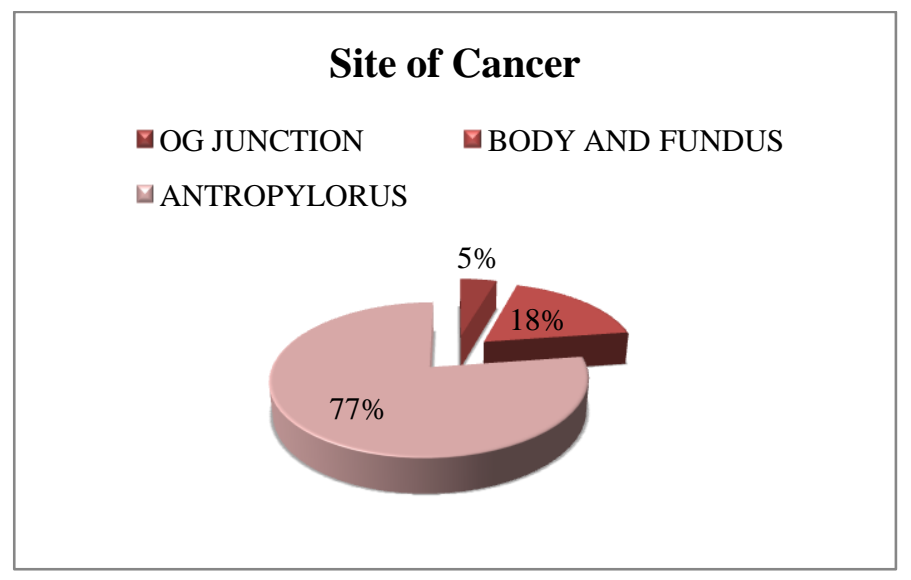

Fig 5: Site of cancer

\section{Conclusion}

Most patients (75\%) were men, $80 \%$ were older than 50 years. The antropyloric region was the most affected anatomical site- $77 \%$.

Adenocarcinoma Stomach accounted for about 95\% of all cases. Most patients had Moderately Differenciated tumors and Subtotal/Total gastrectomy was the most frequent type of surgery.

The present study demonstrated that gastric adenocarcinoma mainly affected men over the age of 50 years. Most adenocarcinomas were in an Moderately Differentiated stage at the time of diagnosis, a fact requiring more aggressive surgical resection in these cases. These data highlight the urgent need for the implementation of preventive measures and early detection programs of gastric cancer.

\section{References}

1. Bailey and Love Short Practice of Surgery.

2. Textbook of Surgery- Seymour I Schwartz

3. Text book of Surgery - Sabiston

4. Text book of Surery-Fischer

5. Devitta-Oncology

6. Stomach Cancer among Japanese- W. Haenzel, M.Kurihara.

7. Epidemiology of Stomach Cancer $-\mathrm{H}$. Brenner, D.Rothenbacher. 